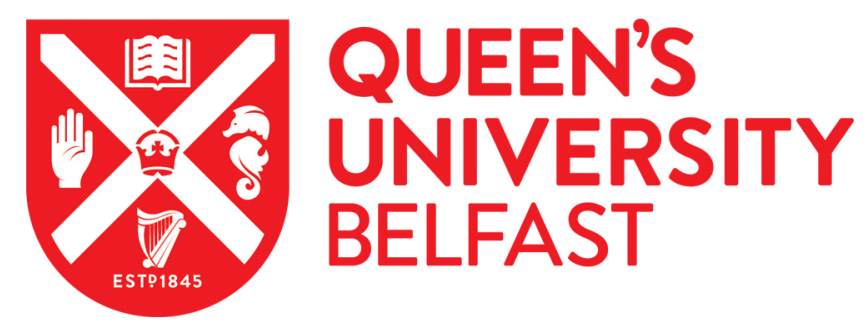

\title{
Space and phase resolved plasma parameters in an industrial dual- frequency capacitively coupled radio-frequency discharge
}

Schulze, J., Gans, T., O'Connell, D., Czarnetzki, U., Ellingboe, A. R., \& Turner, M. M. (2007). Space and phase resolved plasma parameters in an industrial dual-frequency capacitively coupled radio-frequency discharge. Journal of Physics D: Applied Physics, 40 (22)(22), 7008-7018. https://doi.org/10.1088/0022-3727/40/22/022

Published in:

Journal of Physics D: Applied Physics

Queen's University Belfast - Research Portal:

Link to publication record in Queen's University Belfast Research Portal

\section{General rights}

Copyright for the publications made accessible via the Queen's University Belfast Research Portal is retained by the author(s) and / or other copyright owners and it is a condition of accessing these publications that users recognise and abide by the legal requirements associated with these rights.

Take down policy

The Research Portal is Queen's institutional repository that provides access to Queen's research output. Every effort has been made to ensure that content in the Research Portal does not infringe any person's rights, or applicable UK laws. If you discover content in the Research Portal that you believe breaches copyright or violates any law, please contact openaccess@qub.ac.uk. 


\title{
Space and phase resolved plasma parameters in an industrial dual-frequency capacitively coupled radio-frequency discharge
}

\author{
J Schulze ${ }^{1}$, T Gans ${ }^{1,3}$, D O'Connell ${ }^{1,4}$, U Czarnetzki ${ }^{1}$, \\ A R Ellingboe ${ }^{2}$ and $M$ M Turner ${ }^{2}$ \\ ${ }^{1}$ Institute for Plasma and Atomic Physics, Ruhr-University-Bochum, 44780 Bochum, Germany \\ ${ }^{2}$ National Centre for Plasma Science and Technology, Dublin City University, Dublin, Ireland \\ E-mail: fjschulze@hotmail.com
}

Received 8 June 2007, in final form 5 September 2007

Published 2 November 2007

Online at stacks.iop.org/JPhysD/40/7008

\begin{abstract}
The dynamics of high energetic electrons $(\geqslant 11.7 \mathrm{eV})$ in a modified industrial confined dual-frequency capacitively coupled RF discharge (Exelan, Lam Research Inc.), operated at $1.937 \mathrm{MHz}$ and $27.118 \mathrm{MHz}$, is investigated by means of phase resolved optical emission spectroscopy. Operating in a $\mathrm{He}-\mathrm{O}_{2}$ plasma with small rare gas admixtures the emission is measured, with one-dimensional spatial resolution along the discharge axis. Both the low and high frequency RF cycle are resolved. The diagnostic is based on time dependent measurements of the population densities of specifically chosen excited rare gas states. A time dependent model, based on rate equations, describes the dynamics of the population densities of these levels. Based on this model and the comparison of the excitation of various rare gas states, with different excitation thresholds, time and space resolved electron temperature, propagation velocity and qualitative electron density as well as electron energy distribution functions are determined. This information leads to a better understanding of the dual-frequency sheath dynamics and shows, that separate control of ion energy and electron density is limited.
\end{abstract}

(Some figures in this article are in colour only in the electronic version)

\section{Introduction}

Dual-frequency capacitively coupled RF discharges are frequently used in technological applications. They are often applied to etching processes as one of many steps in the production chain of integrated circuits. The advantage of these discharges is separate control of ion energy and ion flux impinging on the substrate surface [1-4]. For this capacitivetype plasma sheath the basic idea is that the high frequency is the dominant contributor to the current and the low frequency

3 Present address: Centre for Plasma Physics, Queen's University Belfast, UK.

4 Present address: Institute for Electrical Engineering and Plasma Technology, Ruhr-University-Bochum, Bochum, Germany. is the dominant contributor to the voltage. The electron density and current is determined by the higher frequency, since electrons can follow the fast potential variations, whereas the much heavier ions can only react to the low frequency. The amplitude of the externally applied low frequency voltage is much higher than the one of the high frequency. Thus, the ion energy, which is determined by the sheath voltage, is influenced mainly by the low frequency component. The RF plasma current, carried by the electrons, determines the power deposition into the plasma through ohmic and sheath heating, and thus the plasma density. The plasma density, in turn, is proportional to the ion (Bohm-like) flux. The ions dominantly react to the time-averaged field, and strike the surface with energy proportional to the RF voltage. 
Space and phase resolved plasma parameters in dual-frequency CCRF discharge

Recent investigations [5-7] have shown that the separate control of electron density and ion energy is limited due to the coupling of both frequencies. However, these studies lack experimental verification. In particular there is minimal investigation of the electron dynamics within the RF cycle [8] in a dual-frequency CCRF discharge.

The key parameter for understanding basic discharge dynamics is the electron energy distribution function (EEDF). It determines dissociation, excitation and ionization processes. Temporal changes of the high energy tail of the EEDF in single frequency RF discharges within the RF cycle have been previously observed by time resolved measurements [9-14]. Phase resolved optical emission spectroscopy (PROES) has demonstrated sensitivity to such phenomena with high spatial and temporal resolution. PROES is non-intrusive and sensitive to plasma parameters like electron density and drift velocity (high energetic electrons), electron temperature and energy distribution functions [12-15]. It is sensitive to the dynamics of high energetic electrons ( $E \geqslant 11.7 \mathrm{eV}$ ) and yields information on these plasma parameters with high temporal resolution on a nanosecond timescale and one-dimensional spatial resolution along the discharge axis. As the spatial and temporal evolution of excitation dynamics of high energetic electrons is particularly relevant for this work, PROES was chosen as diagnostics.

Since only transitions from certain excited levels can be observed, an analytical model of excitation and de-excitation, based on rate equations for the observed levels, is needed in order to determine plasma parameters. In contrast to the standard corona model, based on balance equations and commonly used for optical emission spectroscopy (OES), a complex time dependent model is needed in order to obtain temporally resolved information. This analytical model for the population dynamics within one RF cycle and the results of its application to the measured phase and space resolved emission in the Exelan in terms of electron temperature, drift velocity, density (the diagnostic is only sensitive to high energetic electrons) and EEDF are discussed below.

\section{Experimental setup}

The reactor used for these investigations is a modified industrial dual-frequency CCRF discharge with plane parallel electrodes separated by a gap of $12 \mathrm{~mm}$ (see figure 1). A true summation of the two RF voltages is applied to the bottom electrode, whereas the top electrode is grounded.

The plasma boundary conditions are as follows. The two electrodes are made from single-crystal silicon of radius $110 \mathrm{~mm}$. The upper electrode is water cooled. This is necessary for the reproducibility of industrial processes and investigations of excitation dynamics within the RF cycle, since a stable discharge is needed for several hours. The lower electrode consists of a $200 \mathrm{~mm}$ diameter bare silicon wafer clamped to an electrostatic chuck $(700 \mathrm{~V})$. The gap between the wafer and chuck is filled with helium at 10 Torr to improve heat transfer. An annular silicon ring lies co-planar and immediate outside of the wafer, completing the lower electrode to a total radius of $110 \mathrm{~mm}$. The plasma is confined in the radial direction by adjustable quartz rings $(110 \mathrm{~mm}$ internal dimension), shielding it from the chamber walls. Furthermore,

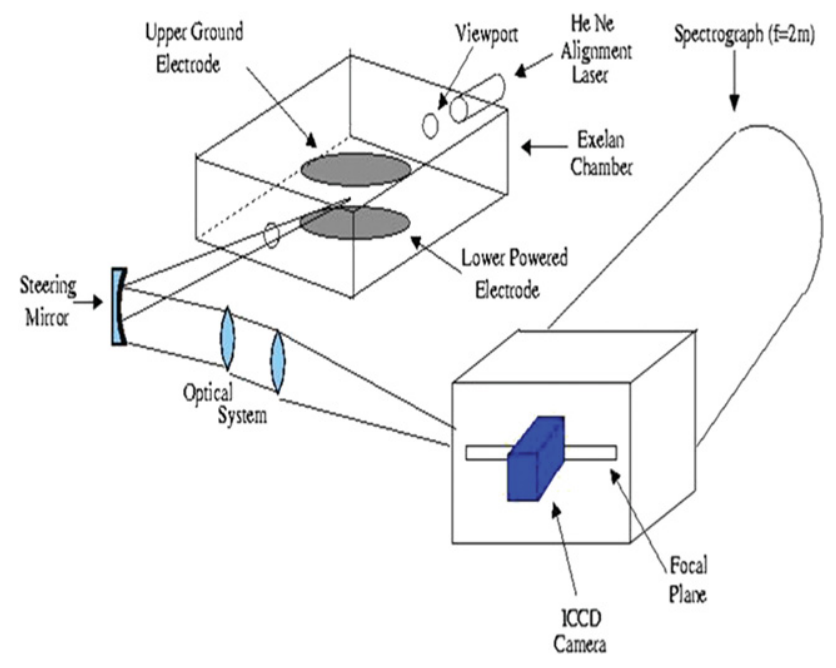

Figure 1. Experimental setup.

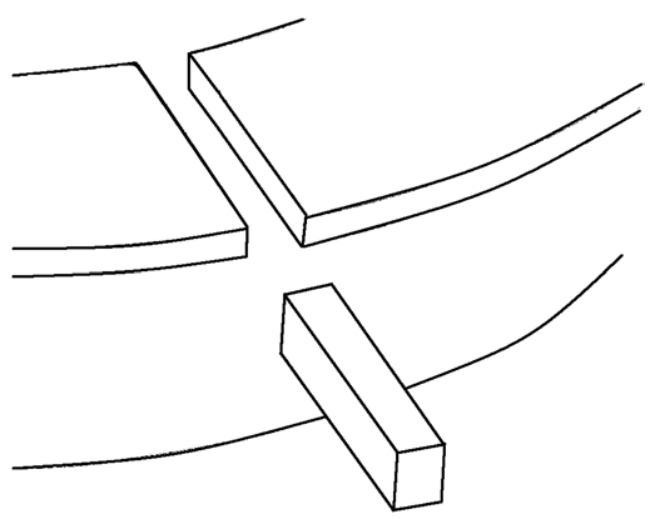

Figure 2. Modification of the confinement rings including the optical block.

the pressure between the rings and the wall is too low, such that no breakdown should occur. Thus, the discharge is almost symmetric. In order to provide access to the chamber for optical diagnostic techniques the original visual access windows were extended and the confinement rings modified. In front of one flange a section of the rings was cut and replaced by an optical quartz block, through which photons can leave the reactor and enter the optical detection system (see figure 2).

Gas is introduced into the chamber through a showerhead built into the upper electrode, and exits the plasma region radially through the confinement rings. The pump-channel outside the confinement rings connects to a high-conductance manifold of a Osaka TC440 helical groove pump, backed by a two stage rotary pump. The pressure drop across the confinement rings results in plasma formation between the electrodes, without breakdown outside the confinement rings, and thus in a nearly symmetric discharge.

For these experiments, the discharge is operated at $P_{27}=800 \mathrm{~W}$ and $P_{2}=200 \mathrm{~W}$ at $p=65 \mathrm{~Pa}$. The RF signals are fixed in frequency at 1.937 and $27.118 \mathrm{MHz}$ with a common phase reference $\left(f_{\mathrm{hf}}=14 \cdot f_{\mathrm{lf}}\right)$. The gas mixtures are He (72\%) and $\mathrm{O}_{2}(19 \%)$ with a $9 \%$ admixture of tracer gases (Ne, Ar, Kr) used as reference gases for PROES. Helium is chosen as the main constituent, as it does not sputter silicon and is the lightest rare gas, for which most data is known. 


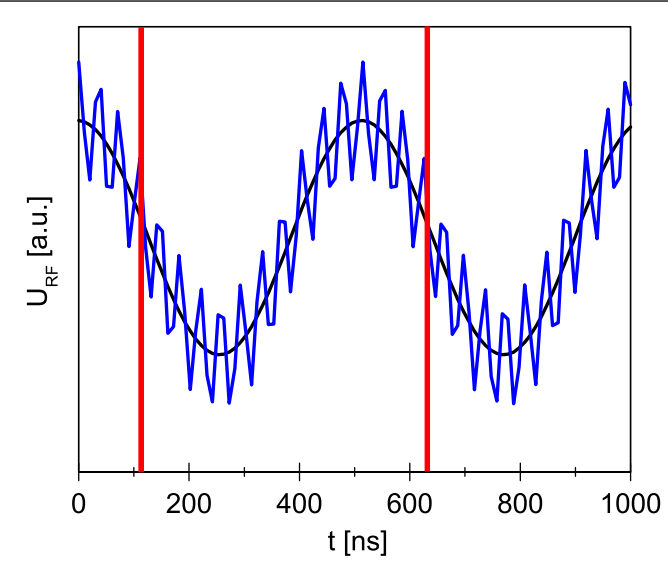

Figure 3. Realization of phase resolved measurements.

Oxygen was used in order to avoid deposition of silicon on the optical block as it reacts with the sputtered particles resulting in quartz-like (transparent) deposition. Furthermore, it stabilizes the discharge, because a pure He discharge is difficult to ignite due to its high ionization energy.

The plasma emission is focused on the entrance slit of a Carl Zeiss PGS 2 spectrometer (300-900 nm) by a lens. The variable slit width was set to $50 \mu \mathrm{m}$ for this experiment. The resolution is $0.00818 \mathrm{~nm} /$ pixel on the camera resulting in $4 \mathrm{~nm} / 512$ pixels. In the spectrograph the light is dispersed and then detected by a fast gateable ICCD camera (PicoStar $\mathrm{HR}, \mathrm{LaVision}$ ), that is triggered and synchronized with the low frequency RF signal (see figure 3). Using this camera, gate widths of a few 100 ps can be realized. In this work the minimum temporal gate width used for phase resolved measurements was $4.1 \mathrm{~ns}$. During this time interval signal is accumulated on the CCD (vertical lines in figure 3). The chosen gate width was a compromise between resolution, signal intensity and acquisition time. The most important feature of the camera is its high repetition rate, maximum $100 \mathrm{MHz}$. Considering the low frequency of $1.937 \mathrm{MHz}$, every RF cycle could be used for phase resolved measurements.

\section{Electron dynamics}

In order to determine plasma parameters from the measured emission an analytical model is applied to the measured data. The standard corona model is based on balance equations and usually used for OES in the case of low density plasmas. However, in order to take into account the transient character of high energetic electrons, an extended, time dependent model based on rate equations must be applied in the case of PROES.

Under the conditions used in this experiment some justified assumptions can be made: the population of the observed level $i$ due to stepwise excitation by electron impact out of excited levels can be neglected. The observed levels were chosen, such that population due to excitation out of metastable levels is generally low. Furthermore, quenching in the pressure range, where the experiment was operated $(65 \mathrm{~Pa})$, and quenching particularly with molecular gases $\left(\mathrm{O}_{2}\right)$ is very effective [16-18]. Therefore, even in metastable levels the population density is so low, that excitation out of metastables can be neglected. As the population densities of excited states including metastables are generally low, reabsorption of radiation must be taken into account only for the case of the strongly populated ground state. Since the population density of excited states is low and the population density of the ground state is very high, radiation resulting from a transition from a higher state into the ground state is efficiently reabsorbed. Due to this reabsorption another atom is excited from the ground state and effectively no de-excitation takes place. Thus, transition coefficients for transitions into the ground state can be neglected in comparison to others.

As the degree of dissociation is low and the quenching coefficients of molecular gases are usually more than one order of magnitude higher than those of rare gases [16], quenching is dominated by $\mathrm{O}_{2}$ and only these processes are taken into account. Due to the lack of available data the quenching coefficients for $\mathrm{O}_{2}$ were approximated by those of $\mathrm{H}_{2}$, which are usually very similar [16]. The observed emission lines were chosen, such that these levels are not quenched effectively. Hence, this approximation does not significantly influence the final result.

For the qualitative investigations of the excitation dynamics $9 \%$ neon was added to the discharge $(72 \% \mathrm{He}, 19 \%$ $\mathrm{O}_{2}, 65 \mathrm{~Pa}$ ). The emission from the $\mathrm{Ne} 2 \mathrm{p}_{1}$-state was observed, since the cascade contribution to the population density of this state can be neglected [20]. Moreover, $\mathrm{Ne} 2 \mathrm{p}_{1}$ has a short lifetime of only $14.5 \mathrm{~ns}$ [19], which allows access to excitation dynamics within the high frequency RF cycle, since the lifetime is shorter than the length of one cycle ( $36.88 \mathrm{~ns})$.

As cascade contributions can be neglected the population density $n_{i}(t)$ of the $\mathrm{Ne} 2 \mathrm{p}_{1}$ state can be described by the following rate equation:

$$
\frac{\mathrm{d} n_{i}(t)}{\mathrm{d} t}=n_{0} E_{0, i}(t)-A_{i} n_{i}(t) .
$$

In equation (1) $E_{0, i}(t)$ is the electron impact excitation function for this state, $n_{0}$ is the ground state density and $A_{i}$ the effective decay rate that considers reabsorption of radiation and quenching:

$$
A_{i}=\sum_{k} A_{i k} g_{i k}+\sum_{q} k_{q} n_{q} .
$$

In equation (2) reabsorption of radiation is included by introducing the escape factors $g_{i k}$, which reflect the probability of one photon originating from the transition from level $i$ to $k$ to leave the plasma without being reabsorbed. Furthermore, quenching is generally considered by summing all products of the density $n_{q}$ of all collision partners and the corresponding quenching coefficients $k_{q}$. Here only quenching with $\mathrm{O}_{2}$ and reabsorption in the case of the strongly populated ground state are taken into account.

The measured number of photons per unit volume and time $n_{p h, i}(t)$ is given by

$$
n_{p h, i}(t)=A_{i k} n_{i}(t) .
$$

Here $A_{i k}$ is the transition probability of the observed emission.

Substituting $n_{i}(t)$, using equations (3) and (1) the excitation into the $\mathrm{Ne} 2 \mathrm{p}_{1}$ state can be directly determined out of the measured emission by the following equation:

$$
E_{i}(t)=\frac{1}{A_{i k} n_{0}}\left(\frac{\mathrm{d} n_{p h, i}(t)}{\mathrm{d} t}+A_{i} n_{p h, i}(t)\right) .
$$




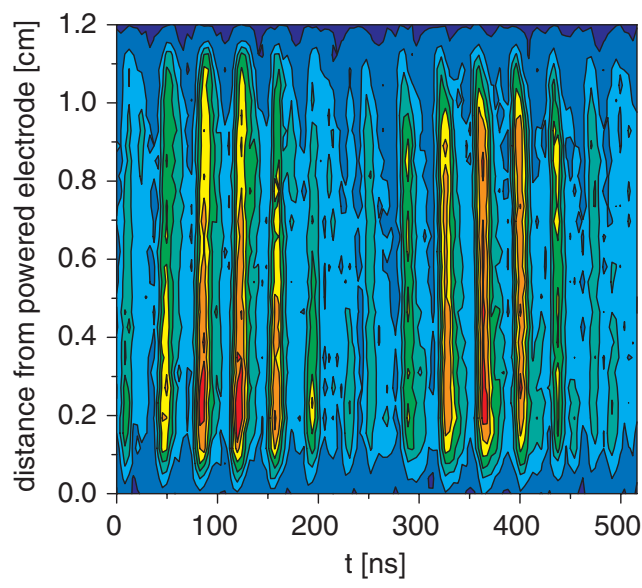

Figure 4. Phase and space resolved electron impact excitation function of $\mathrm{Ne} 2 \mathrm{p}_{1}\left(\Phi_{\mathrm{He}}=2175 \mathrm{sccm}, \Phi_{\mathrm{O}_{2}}=397 \mathrm{sccm}, \Phi_{\mathrm{Ne}}=\right.$ $\left.292 \mathrm{sccm}, p=65 \mathrm{~Pa}, P_{27}=800 \mathrm{~W}, P_{2}=200 \mathrm{~W}\right)$. Red corresponds to high and blue to low excitation. (Colour online.)

This direct access to the excitation function is possible in the case of the $\mathrm{Ne} 2 \mathrm{p}_{1}$ state, since cascade contributions can be neglected.

Figure 4 displays the phase and space resolved excitation of the $\mathrm{Ne} 2 \mathrm{p}_{1}$-state within one low frequency $\mathrm{RF}$ cycle resolving every high frequency cycle (abscissa) with one-dimensional spatial resolution along the discharge axis (ordinate). In this figure (in colour online) the colour scale is chosen such that red corresponds to high excitation and blue to low excitation. An overall structure of four excitation maxima [7], two at each electrode, and a fine structure of fourteen submaxima can be identified. The excitation maxima are caused by the rapid oscillations of the sheath edge, mainly determined by the high frequency component. However, the velocity of these oscillations depends on the spatial movements of the sheath edge and, therefore, on the local ion-density. The spatial structure of the sheath is mainly determined by the large low frequency voltage. Consequently, the excitation is also influenced by the low frequency component and there is a strong coupling between both frequencies.

Figures 5 and 6 show sections of figure 4 during the first and second half of one low frequency RF cycle close to the top and bottom electrode, respectively. The time dependence of the excitation in the discharge under these conditions can be described by the square of a sum of two sinusoidal functions, one corresponding to the high and the other to the low frequency voltage:

$$
E(t)=\left(C_{\mathrm{lf}} \sin \left(\omega_{\mathrm{lf}} t+\Phi_{\mathrm{lf}}\right)+C_{\mathrm{hf}} \sin \left(\omega_{\mathrm{hf}} t+\Phi_{\mathrm{hf}}\right)\right)^{2}+B
$$

In equation (5) the amplitudes $C_{\mathrm{lf}}$ and $C_{\mathrm{hf}}$ mirror the contribution of the respective frequency to the overall excitation. $\Phi_{\mathrm{lf}}$ and $\Phi_{\mathrm{hf}}$ are phase shifts and B is a temporally constant background. The values of these parameters depend on the spatial position, the respective half of the low frequency RF cycle and the emission line. Equation (5) is a mathematical function, that reproduces the measured temporal excitation profiles. This mathematical description of the temporal excitation profile is needed for the later determination of

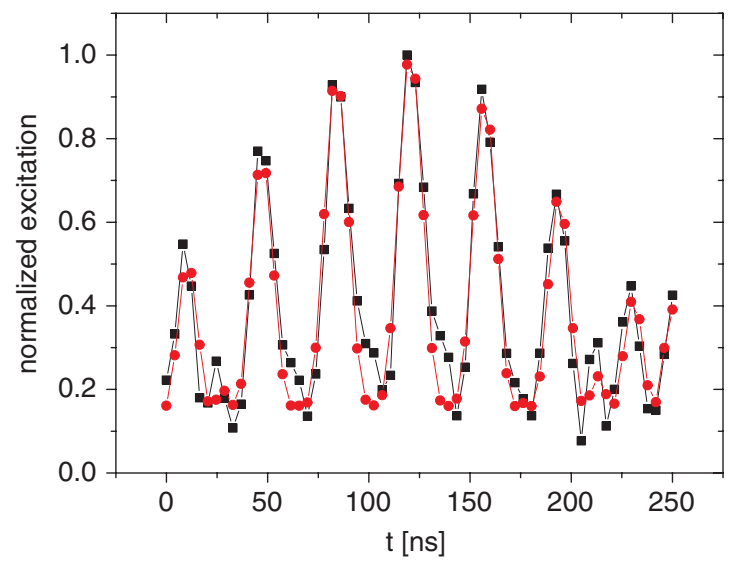

Figure 5. Comparison between modelled (red line/dots, equation (5)) and calculated (black line/squares, equation (5)) excitation of $\mathrm{Ne} 2 \mathrm{p}_{1}$ close to the bottom electrode sheath edge $(y=2.3 \mathrm{~mm})$ during the first half of one low frequency RF cycle. (Colour online.)

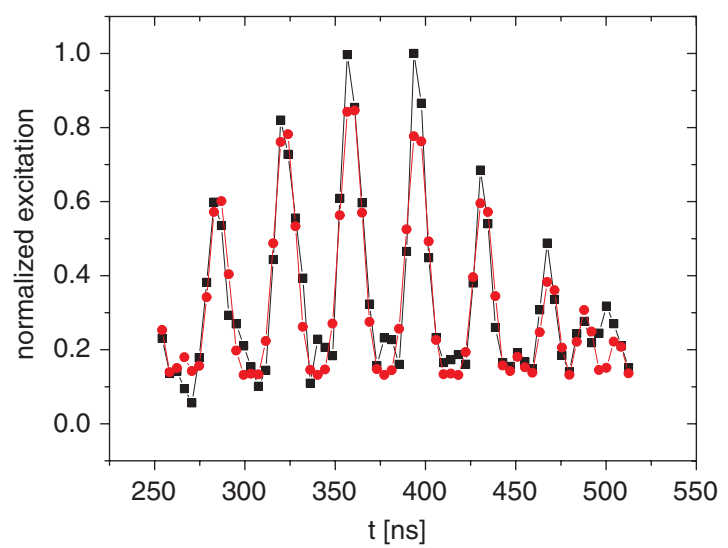

Figure 6. Comparison between modeled (red line/dots, equation (5)) and calculated (black line/squares, equation (5)) excitation of $\mathrm{Ne} 2 \mathrm{p}_{1}$ close to the top electrode sheath edge $(y=2.3 \mathrm{~mm})$ during the second half of one low frequency RF cycle. (Colour online.)

EEDFs and plasma parameters. In general, the excitation into all observed levels is assumed to be well described by the shape of equation (5), since the excitation mechanism is the same for all levels and only depends on the discharge. Only the amplitudes $C_{\mathrm{lf}}, C_{\mathrm{hf}}$ as well as the temporally constant background $B$ are different for each energy state.

\section{Population dynamics of excited states}

As explicit plasma parameters can only be determined out of the comparison of different emission lines, of different rare gas admixtures, energy states, for which contributions due to cascades cannot be neglected, had to be used. The population density $n_{i}(t)$ of the investigated state $i$ can be described by the following rate equation, which takes into account cascades from level $c$ :

$$
\frac{\mathrm{d} n_{i}(t)}{\mathrm{d} t}=n_{0} E_{0, i}(t)+A_{\mathrm{c} i} n_{\mathrm{c}}(t)-A_{i} n_{i}(t) .
$$

In contrast to the $\mathrm{Ne} 2 \mathrm{p}_{1}$ state a direct access to the excitation function is no longer possible. In order to solve equation (6) for the population density $n_{i}(t)$, the population 
Table 1. Table of emission lines, that are used for the investigation of electron dynamics, including the characteristic data of each line.

\begin{tabular}{lllllll}
\hline State & $\begin{array}{l}\lambda[19] \\
(\mathrm{nm})\end{array}$ & $\begin{array}{l}\mathrm{E}[19] \\
(\mathrm{eV})\end{array}$ & $\begin{array}{l}\tau \\
(\mathrm{ns})\end{array}$ & $\begin{array}{l}A_{i k} \\
\left(\frac{1}{s}\right)\end{array}$ & $\begin{array}{l}k \\
\left(10^{-10} \mathrm{~cm}^{3} \mathrm{~s}^{-1}\right)\end{array}$ & $C / D$ \\
\hline $\mathrm{Kr} 2 \mathrm{p}_{5}$ & 758.7 & 11.7 & $21.5[13]$ & 46511628 & $1.7[13]$ & $0.3[22]$ \\
$\mathrm{Ar} 2 \mathrm{p}_{1}$ & 750.4 & 13.5 & $34.1[19]$ & 44642857 & $0.3[23]$ & $0.2[21]$ \\
$\mathrm{Ne} 2 \mathrm{p}_{1}$ & 585.2 & 19.0 & $14.5[19]$ & 68275862 & $2.3[13]$ & $0.03[20]$ \\
\hline
\end{tabular}

density $n_{\mathrm{c}}(t)$ of the dominant cascade level must be known. In order to achieve this, the rate equation of the dominant cascade level must be solved:

$$
\begin{gathered}
\frac{\mathrm{d} n_{\mathrm{c}}(t)}{\mathrm{d} t}=n_{0} E_{0, \mathrm{c}}(t)-A_{\mathrm{c}} n_{\mathrm{c}}(t), \\
n_{i, \mathrm{c}}(t)=n_{i, \mathrm{c}}\left(t+T_{\mathrm{RF}}\right) .
\end{gathered}
$$

In equation (8), $T_{\mathrm{RF}}$ is the length of one low frequency RF cycle. As the contribution of cascades to the population densities of the states used in this work is generally low (see table 1), second order cascades can be neglected in equation (7). The solution $n_{i}(t)$ is [12]

$$
\begin{aligned}
n_{i}(t) & =\left(\frac{n_{0} \tilde{E}_{i}\left(T_{\mathrm{RF}}, A_{i}\right) \mathrm{e}^{-A_{i} T_{\mathrm{RF}}}}{1-\mathrm{e}^{-A_{i} T_{\mathrm{RF}}}}+n_{0} \tilde{E}_{i}\left(t, A_{i}\right)\right) \mathrm{e}^{-A_{i} t} \\
& +\frac{A_{\mathrm{c} i}}{A_{i}-A_{\mathrm{c}}}\left[\left(\frac{n_{0} \tilde{E}_{\mathrm{c}}\left(T_{\mathrm{RF}}, A_{\mathrm{c}}\right) \mathrm{e}^{-A_{\mathrm{c}} T_{\mathrm{RF}}}}{1-\mathrm{e}^{-A_{\mathrm{c}} T_{\mathrm{RF}}}}+n_{0} \tilde{E}_{\mathrm{c}}\left(t, A_{\mathrm{c}}\right)\right) \mathrm{e}^{-A_{\mathrm{c}} t}\right. \\
& \left.-\left(\frac{n_{0} \tilde{E}_{\mathrm{c}}\left(T_{\mathrm{RF}}, A_{i}\right) \mathrm{e}^{-A_{i} T_{\mathrm{RF}}}}{1-\mathrm{e}^{-A_{i} T_{\mathrm{RF}}}}+n_{0} \tilde{E}_{\mathrm{c}}\left(t, A_{i}\right)\right) \mathrm{e}^{-A_{i} t}\right]
\end{aligned}
$$

with

$$
\tilde{E}_{x}\left(t, A_{y}\right)=\int_{0}^{t} E_{x}\left(t^{\prime}\right) \mathrm{e}^{A_{y} t^{\prime}} \mathrm{d} t^{\prime} .
$$

Using equation (3) the population density, calculated by equation (9), can be fitted to the measured emission by varying the free parameters $C_{\mathrm{lf}}, C_{\mathrm{hf}}, \Phi_{\mathrm{lf}}, \Phi_{\mathrm{hf}}$ and $B$ in equation (5) at each position in the discharge. As the emission during the first half of the low frequency RF cycle is caused by high energetic electrons, that are accelerated by the bottom electrode's sheath, whereas the emission during the second half is caused by electrons from the top electrode and as the discharge is slightly asymmetric, this fit is performed separately for the first and second half individually at each position in the discharge. The fact, that electrons are accelerated by different sheaths during different halves of one low frequency RF cycle, causes $C_{\text {lf }}$ and $C_{\mathrm{hf}}$ to be substantially different at a given spatial position during the two different halves for a certain energy level. If the discharge was perfectly symmetric, $E(t, z)=E\left(\frac{T}{2}+t, L-z\right)$ would hold. Here $T$ is the length of one low frequency RF cycle, $L$ the electrode gap, $t$ a variable time and $z$ a variable position in the discharge.

Once this fit is completed, the free parameters are determined and the excitation function $E_{i}(t)$ is calculated at a specific location in the discharge chamber using equation (5).

\section{Determination of phase and space resolved EEDFs}

The three states listed in table 1 were used to determine EEDFs.

In table $1, C / D$ denotes the contribution of cascades in relation to direct excitation of the corresponding state as they were determined from electron beam experiments [20-22]. The relatively small contribution of cascades justifies the neglect of second order cascades. The excitation thresholds of these states cover an energy interval from 11.7 to $19.0 \mathrm{eV}$. The corresponding electron impact excitation cross sections are accurately known from [20-22].

Once the excitation functions of each line are known, by applying the time and space resolved techniques, which is described in the previous section, the EEDF can be determined at high temporal and spatial resolution. The relation between excitation function $E_{i}$ and $\operatorname{EEDF} f(E)$ is

$$
E_{\mathrm{i}}=n_{\mathrm{e}} \int_{0}^{\infty} \sigma_{\mathrm{i}}(E) \sqrt{\frac{2 E}{m_{\mathrm{e}}}} f(E) \mathrm{d} E .
$$

Assuming a justified shape of the EEDF in terms of a mathematical function with a set of free parameters, the RHS of equation (11) can be fitted to the corresponding analytical description of the excitation function using equation (5). As only a relative calibration of the optical setup was possible, the factor $n_{\mathrm{e}}$ in equation (11) does not correspond to the absolute electron density but is proportional to it. $m_{\mathrm{e}}$ denotes the electron mass. Fitting the RHS of equation (11) to the analytical description of the excitation, at different locations in the plasma, at different times in the RF cycle, the EEDF, including the free parameters (electron temperature and drift velocity) and the qualitative behaviour of the electron density, can be determined both temporally and spatially resolved.

Since the discharge is capacitive with electric fields perpendicular to the electrodes, a shifted Maxwellian distribution function was chosen, as it takes into account a drift velocity $V_{\mathrm{d}}$ in direction of the electric field:

$$
f_{\text {Drift }}(V)=\left(\frac{m_{\mathrm{e}}}{2 \pi k_{\mathrm{B}} T_{\mathrm{e}}}\right)^{\frac{3}{2}} \mathrm{e}^{-\frac{m_{\mathrm{e}}}{2} \cdot \frac{V_{x}^{2}+V_{y}^{2}+\left(V_{z}-V_{\mathrm{d}}\right)^{2}}{k_{b} T_{\mathrm{e}}}} .
$$

Transformation of the above equation into energy space yields:

$$
f_{\text {Drift }}(E)=\frac{1}{2 \sqrt{\pi k_{\mathrm{B}} T_{\mathrm{e}} E_{\mathrm{d}}}} \mathrm{e}^{-\frac{\left(\sqrt{E}-\sqrt{E_{\mathrm{d}}}\right)^{2}}{k_{\mathrm{B}} T_{\mathrm{e}}}}\left(1-\mathrm{e}^{-\frac{4 \sqrt{E} \sqrt{E_{\mathrm{d}}}}{k_{\mathrm{B}} T_{\mathrm{e}}}}\right)
$$

This equation is later fitted to the measured excitation (equation (11)) varying electron temperature $T_{\mathrm{e}}$ and drift energy $E_{\mathrm{d}}$.

It should be noted, that in equation (13), $T_{\mathrm{e}}$ does not represent an electron temperature in the classical sense, i.e. $k T_{\mathrm{e}} \neq \frac{2}{3}\langle\varepsilon\rangle$, where $\langle\varepsilon\rangle$ is the electron mean energy. Instead, it is assumed, that within the region between the threshold for excitation (about $12 \mathrm{eV}$ ) and well above the highest level $(19 \mathrm{eV})$ the distribution function can be described by equation (13). Therefore, $T_{\mathrm{e}}$ is only a parameter used for the 
description of the distribution function. In the following $T_{\mathrm{e}}$ will simply be called electron temperature. Furthermore, it is assumed, that the determined EEDF is also valid for electrons with energies below the threshold energy. As only energy levels with threshold energies higher than $11.7 \mathrm{eV}$ were used, there could be deviations at lower energies, that cannot be observed with this emission spectroscopic technique.

In order to determine the electron temperature, drift velocity and electron density the following technique is applied to all positions in the discharge and for each half of one low frequency RF cycle separately:

Initially the excitation functions of all three emission lines are determined individually under variation of the amplitudes in equation (5) by fitting the calculated emission, that results out of the model to describe the population density of an excited state (see section 4), to the measured emission characteristics. The electron temperature is then determined from the ratio of the excitation functions of the $\mathrm{Ne} 2 \mathrm{p}_{1}$ - and $\mathrm{Kr} 2 \mathrm{p}_{5}$-state at each position, for each half of one cycle individually. At the phases of minimum excitation in one low frequency RF cycle the drift velocity is assumed to be low. Therefore, the distribution of electrons is approximately Maxwellian. Assuming such a Maxwellian distribution at the phases of minimum excitation, the ratio of the excitation functions of two emission lines only depends on the electron temperature $T_{\mathrm{e}}$. Using equation (11), $n_{\mathrm{e}}$ cancels in the ratio:

$$
\frac{E_{\mathrm{Ne} 2 \mathrm{p}_{1}}}{E_{\mathrm{Kr} 2 \mathrm{p}_{5}}}=\frac{\int \sigma_{\mathrm{Ne} 2 \mathrm{p}_{1}} \sqrt{\frac{2 E}{m_{\mathrm{e}}}} 2 \sqrt{\frac{E}{\pi\left(k_{\mathrm{B}} T_{\mathrm{e}}\right)^{3}}} \mathrm{e}^{-\frac{E}{k_{\mathrm{B}} T_{\mathrm{e}}}} \mathrm{d} E}{\int \sigma_{\mathrm{Kr} 2 \mathrm{p}_{5}} \sqrt{\frac{2 E}{m_{\mathrm{e}}}} 2 \sqrt{\frac{E}{\pi\left(k_{\mathrm{B}} T_{\mathrm{e}}\right)^{3}}} \mathrm{e}^{-\frac{E}{k_{\mathrm{B}} T_{\mathrm{e}}}} \mathrm{d} E}
$$

As the ratio on the LHS of equation (14) is known from the analytical description of the individual excitation functions, equation (14) yields the electron temperature at the corresponding phases. Since the temperatures at the individual phases never differ from each other by more than $5 \%$, the electron temperature is assumed to be temporally constant in each half of the low frequency cycle. Figure 7 shows the ratio of both excitation functions as a function of $T_{\mathrm{e}}$. One can clearly see that the determination of the electron temperature, using this approach, is unique.

Once the electron temperature $T_{\mathrm{e}}$ has been determined, it is assumed to be temporally constant at the given position in the plasma for the given half of the RF cycle. In the final step the excitation functions of all three lines are fitted simultaneously to the respective analytically determined excitation function varying $v_{\mathrm{d}}$ and $n_{\mathrm{e}}$ in equation (11) and assuming a shifted Maxwellian distribution function (equation (13)). Such a fit is performed simultaneously for all lines at each phase in the low frequency RF cycle at a given position in the discharge. The result of the drift velocity and electron density at each phase is unique, since the ratio of two excitation functions of different states depends only on $T_{\mathrm{e}}$ and $V_{\mathrm{d}}$ ( $n_{\mathrm{e}}$ cancels). As $T_{\mathrm{e}}$ has already been determined by the above described technique, the ratio only depends on the drift velocity. Figure 8 shows a plot of the ratio of the excitation function of $\mathrm{Ne} 2 \mathrm{p}_{1}$ and $\mathrm{Kr} 2 \mathrm{p}_{5}$ as a function of electron temperature and drift velocity. In addition to $\mathrm{Ne} 2 \mathrm{p}_{1}$ and $\mathrm{Kr} 2 \mathrm{p}_{5} \operatorname{Ar} 2 \mathrm{p}_{1}$ is used as a third emission line in order to improve statistics. The result is a surface, which is, in case of a fixed temperature, reduced to a

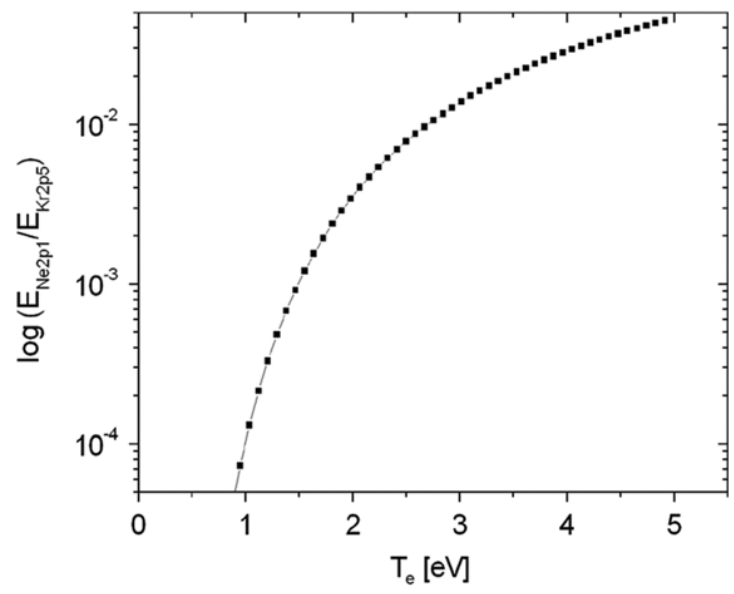

Figure 7. Semi-logarithmic plot of the ratio of the excitation functions of $\mathrm{Ne} 2 \mathrm{p}_{1}$ and $\mathrm{Kr} 2 \mathrm{p}_{5}$ as a function of the electron temperature assuming a Maxwellian distribution function.

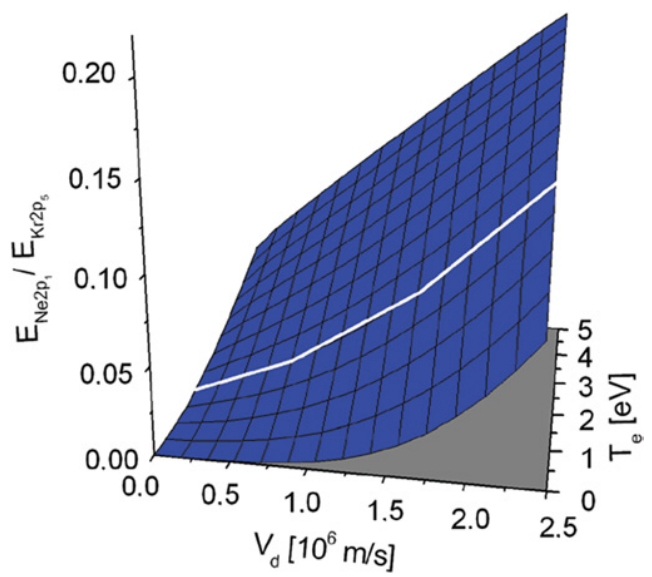

Figure 8. Ratio of the excitation functions of $\mathrm{Ne} 2 \mathrm{p}_{1}$ and $\mathrm{Kr} 2 \mathrm{p}_{5}$ as a function of drift velocity and electron temperature assuming a shifted Maxwellian distribution function. The line indicates the fact, that the ratio only depends on $v_{\mathrm{d}}$ at a given $T_{\mathrm{e}}$.

strictly increasing line (indicated line in figure 8). Knowing the ratio, the determination of $V_{\mathrm{d}}$ is unique. $n_{\mathrm{e}}$ directly results from knowing $V_{\mathrm{d}}$ and $T_{\mathrm{e}}$ and is, therefore, also unique.

An example for the determination of $V_{\mathrm{d}}$ and $n_{\mathrm{e}}$ at $y=$ $0.23 \mathrm{~cm}$ and $t=364.9 \mathrm{~ns}$ by a least square fit is shown in figure 9. At this phase there is maximum excitation in the second half of the RF cycle close to the bottom electrode. Figure 9 shows a logarithmic plot of $\chi^{2}$ as a function of $E_{\mathrm{d}}$ and $n_{\mathrm{e}}$. Here $\chi^{2}$ is defined as $\chi^{2}=\left(E_{\mathrm{an}}-E_{\mathrm{fit}}\right)^{2} / \sigma^{2}$, where $E_{\mathrm{an}}$ is the analytically determined value for the excitation (see section 4) and $E_{\text {fit }}$ the calculated value for the excitation resulting from the fit routine described in this paper. $\sigma$ is the error, that occurs in terms of the analytical determination of the excitation (typically $5 \%$ of $E_{\text {an }}$ ). One can observe a well defined minimum at $E_{\mathrm{d}} \approx 4.1 \mathrm{eV}\left(V_{\mathrm{d}} \approx 1.2 \times 10^{6} \mathrm{~m} \mathrm{~s}^{-1}\right)$ and $n_{\mathrm{e}} \approx 0.3$. This minimum again shows, that the result of this fit is unique. For instance, the minimal $\chi^{2}$ at $E_{\mathrm{d}}=0$ corresponding to a Maxwellian distribution function is more than two orders of magnitude higher than the minimum at $E_{\mathrm{d}} \approx 4.1 \mathrm{eV}$. Further details regarding this algorithm are mentioned in the appendix. 
Figure 10 summarizes the model to determine the EEDF including drift velocity, electron temperature and electron density. An analytical description for the electron impact excitation function is found to be $E(t)=\left(C_{\mathrm{lf}} \sin \left(\omega_{\mathrm{lf}} t+\Phi_{\mathrm{lf}}\right)+\right.$ $\left.C_{\mathrm{hf}} \sin \left(\omega_{\mathrm{hf}} t+\Phi_{\mathrm{hf}}\right)\right)^{2}+B\left[(1)\right.$, Ne $2 \mathrm{p}_{1}$-state, no cascades $]$. Varying the free parameters in this analytical description, the excitation function of each state is determined individually by fitting the emission calculated out of the expression for the population density ((2a), equation (9)) of the respective state to the measured emission (2b). The electron temperature is determined for each location individually out of the ratio of two excitation functions assuming a Maxwellian EEDF at the phases of minimum excitation [(3), equation (14)]. $V_{\mathrm{d}}$ and $n_{\mathrm{e}}$ are then varied fitting the excitation calculated out

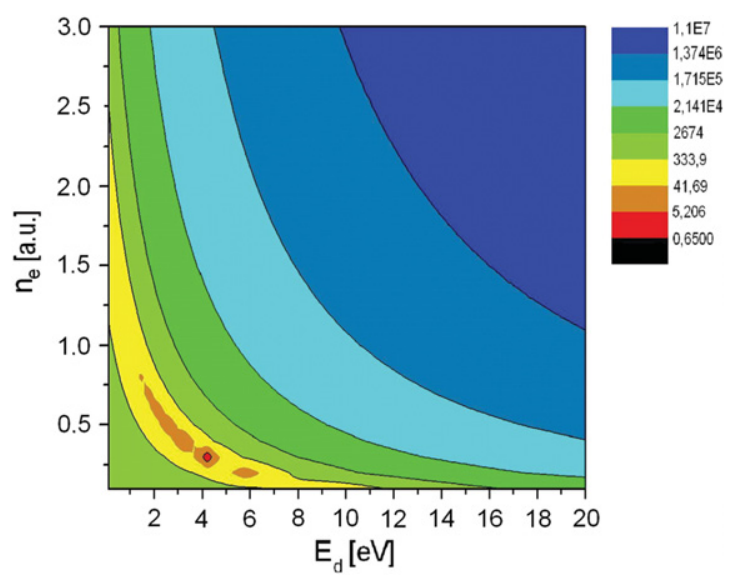

Figure 9. Logarithmic plot of $\chi^{2}$ as a function of $E_{\mathrm{d}}$ and $n_{\mathrm{e}}$ at $y=2.3 \mathrm{~mm}$ and $t=364.9 \mathrm{~ns}$. (Colour online.) of the EEDF (equation (11)) to the analytically determined one (4).

\section{Results}

Typical examples of EEDFs close to the powered bottom electrode at the phases of maximum and minimum excitation as well as an intermediate phase are shown in figure 11 . The EEDF is approximately purely Maxwellian (no drift component) at phases of minimum excitation and strongly shifted at phases of maximum excitation. This clearly demonstrates the strong influence of the drift component.

Examples for the obtained parameter sets, that describe the EEDF, are shown in terms of the following plots.

Figure 12 shows the time and space resolved electron drift velocity in $\mathrm{m} \mathrm{s}^{-1}$ in the discharge. It is maximum at phases of maximum excitation and minimum at phases of minimum excitation. The maximum absolute values in the bulk are of the order of $1.3 \cdot 10^{6} \mathrm{~m} \mathrm{~s}^{-1}$. The absolute maximum of $2 \times 10^{6} \mathrm{~m} \mathrm{~s}^{-1}$ occurs at the sheath edge, where the electrons are accelerated by the expanding sheath. The further these fast electron beams penetrate into the plasma bulk the more energy is lost through collisions with the background gas (heating) and the lower the remaining drift velocity. Generally the electron drift velocity is high at the sheath edge, where the electron beam is generated, decreases in the bulk and increases again as it approaches the opposite electrode. The interaction of energetic electrons with the opposite sheath is complex and the interpretation of this characteristic of the drift velocity is not clear.

Vender and Wood obtained very similar results for the drift velocity of highly energetic electron beams in PIC simulations

(3)

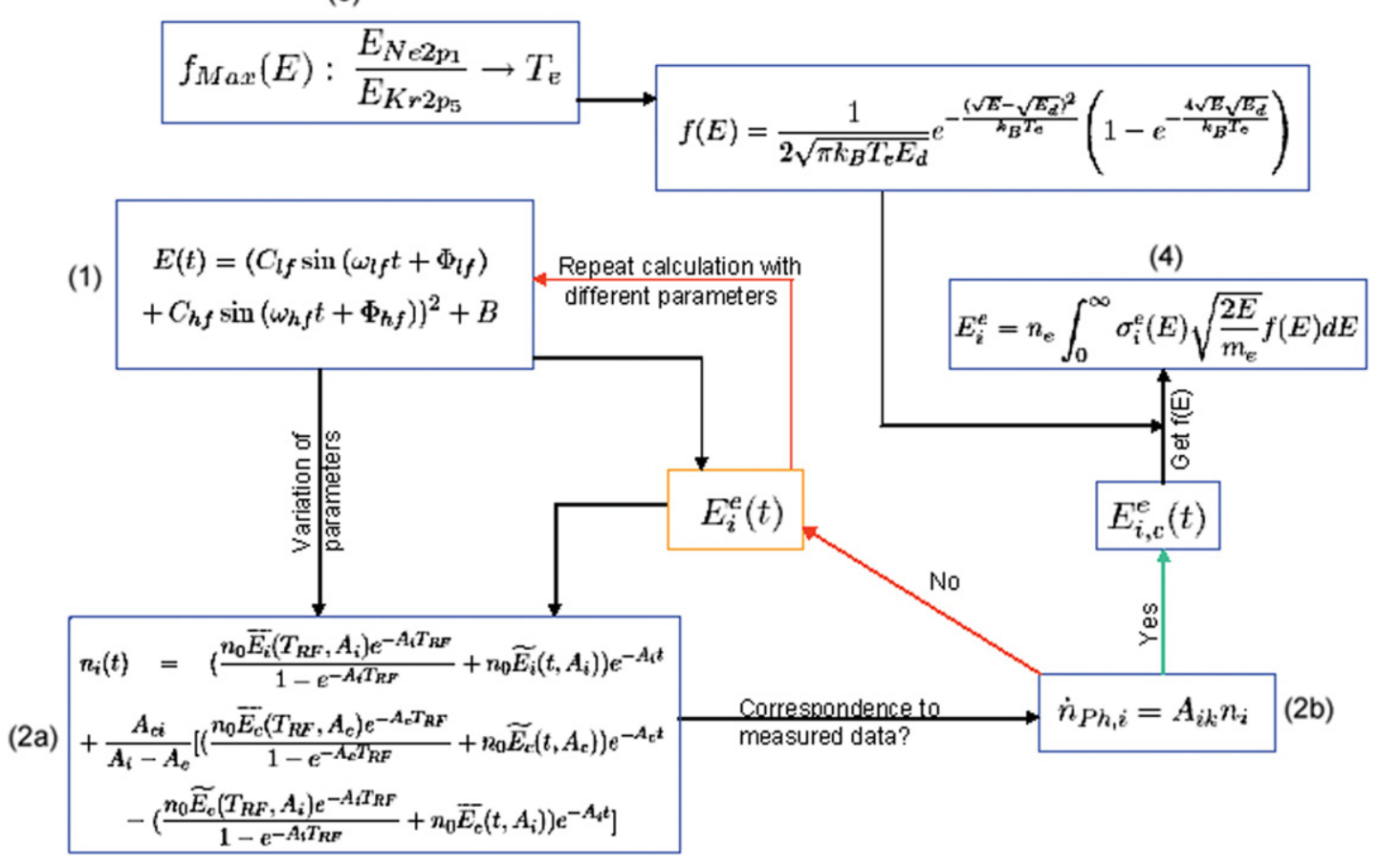

Figure 10. Schematic of the model to determine the EEDF out of the measured emission using several emission lines. 


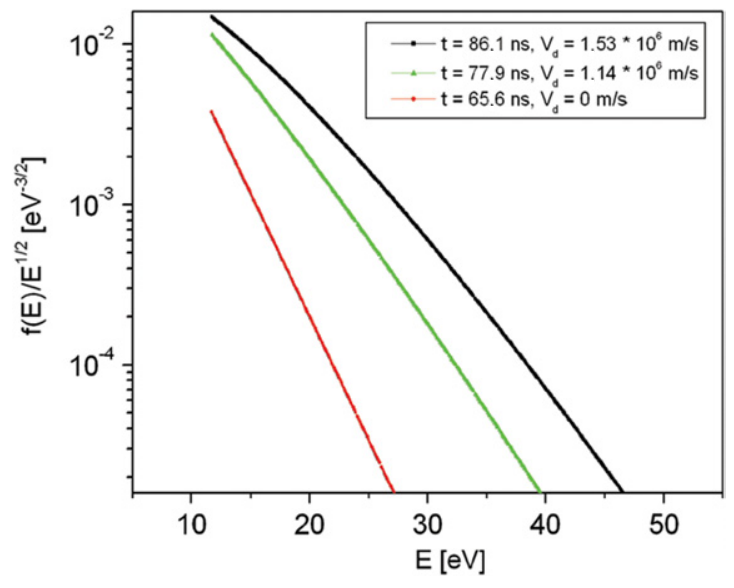

Figure 11. EEDFs close to the bottom electrode sheath edge $(y=2.3 \mathrm{~mm})$ at $t=86.1 \mathrm{~ns}$ (maximum drift velocity $V_{\mathrm{d}} \approx 1.53 \times 10^{6} \mathrm{~m} \mathrm{~s}^{-1}$ ), $t=77.9 \mathrm{~ns}$ and $t=65.6 \mathrm{~ns}$ (minimum drift velocity $V_{\mathrm{d}} \approx 0 \mathrm{~m} \mathrm{~s}^{-1}$ ) at $T_{\mathrm{e}}=2.85 \mathrm{eV}$.

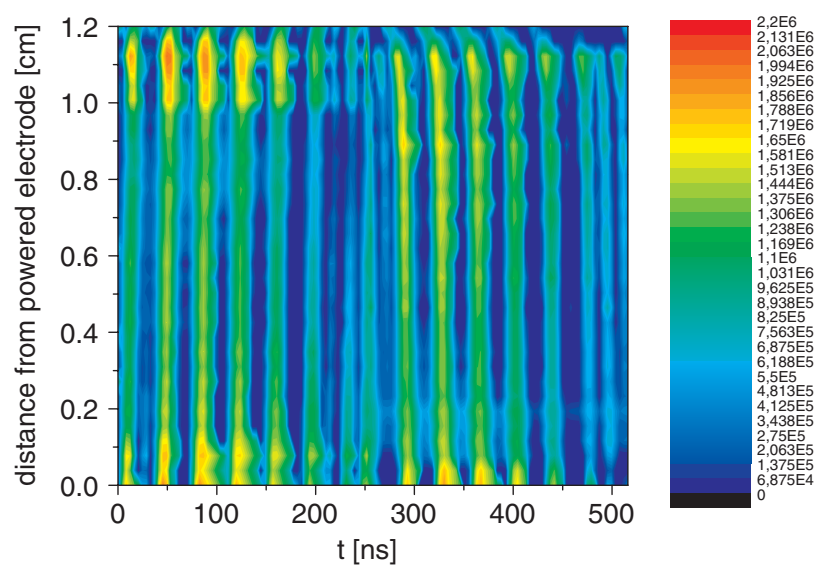

Figure 12. Phase and space resolved contour plot of the electron drift velocity in the discharge. (Colour online.)

of single frequency CCRF discharges in $\mathrm{H}_{2}$ [24] and argon [25] at low pressures, respectively. Gans observed similar drift velocities experimentally in $\mathrm{H}_{2}$ at high pressures [12] and O'Connell observed beam-like highly energetic electrons in $\mathrm{H}_{2}$ at low pressures [26]. All these simulations and experiments show the generation of highly energetic electrons with a drift velocity of the order of $10^{6} \mathrm{~m} \mathrm{~s}^{-1}$ at the sheath edge.

Figures 13 and 14 show the space resolved electron density at the phases of maximum excitation in the plasma bulk during both halves of one low frequency RF cycle, respectively. The density is maximum at the sheath edges, where the drift velocities are maximum. It increases with time as long as confinement of electrons is good. One can observe the collapse of the powered bottom electrode sheath during the first half and of the grounded top electrode sheath during the second half, since the density at the expanded sheath increases with time in relation to the density at the collapsed sheath. The density profiles in figures 13 and 14 are different, since the figures show density profiles during different halves of one low frequency RF cycle. During the first half the low frequency sheath in front of the powered electrode collapses and beams propagate towards the grounded electrode, where they hit the sheath and are reflected. During the second half, the low frequency

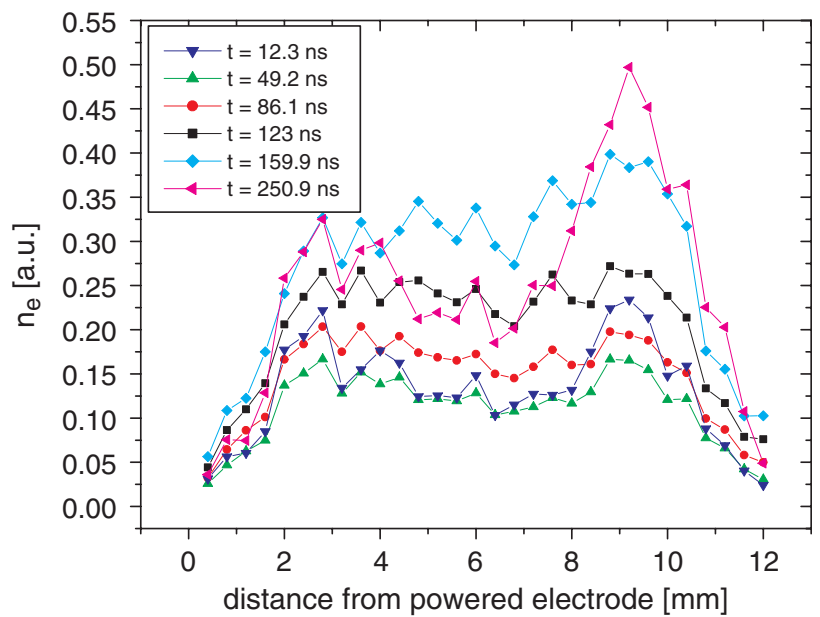

Figure 13. Space resolved electron density $(E \geqslant 11.7 \mathrm{eV})$ at the phases of maximum excitation in the plasma bulk during the first half of one low frequency RF cycle.

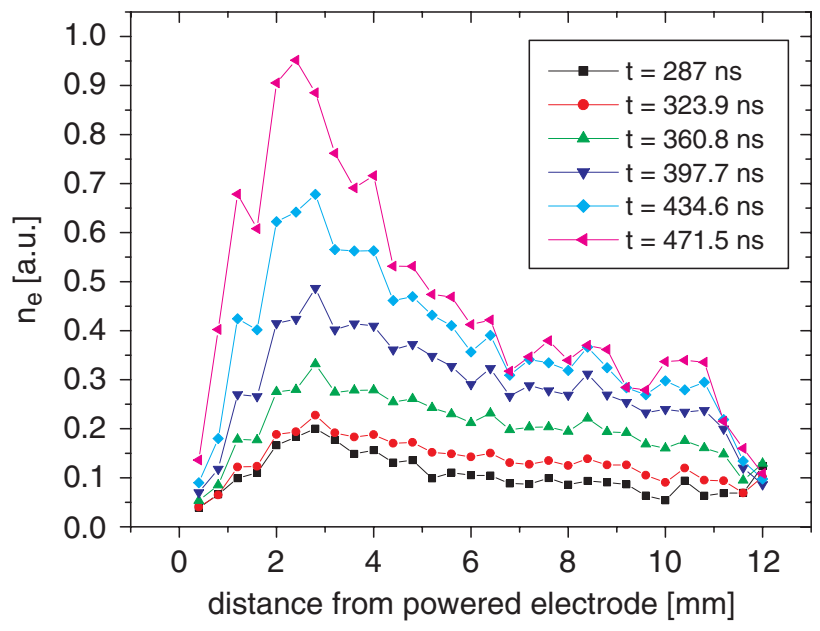

Figure 14. Space resolved electron density $(E \geqslant 11.7 \mathrm{eV})$ at the phases of maximum excitation in the plasma bulk during the second half of one low frequency RF cycle.

sheath in front of the grounded electrode collapses, beams propagate towards the powered electrode and are reflected. The density always peaks close to the sheath edge of the expanded low frequency sheath (at the grounded electrode during the first half and at the powered electrode during the second half). This is caused by three different mechanisms: considering the first half of one low frequency RF cycle, electrons are accelerated towards the grounded electrode by the expanding high frequency sheath at the powered electrode at phases of collapsed low frequency sheath. The generation of such an electron beam takes place during the time interval of high frequency sheath expansion. As this generation does not happen instantaneously, different beam electrons hit the opposite sheath at different times. The first electrons, that arrive at the opposite sheath, enter the sheath and are reflected. However, it takes some time until these electrons leave the sheath again. When they leave the sheath again, beam electrons propagating in direction towards the grounded electrode arrive at the same position. This increases the density at the grounded electrode in comparison to the powered electrode. 


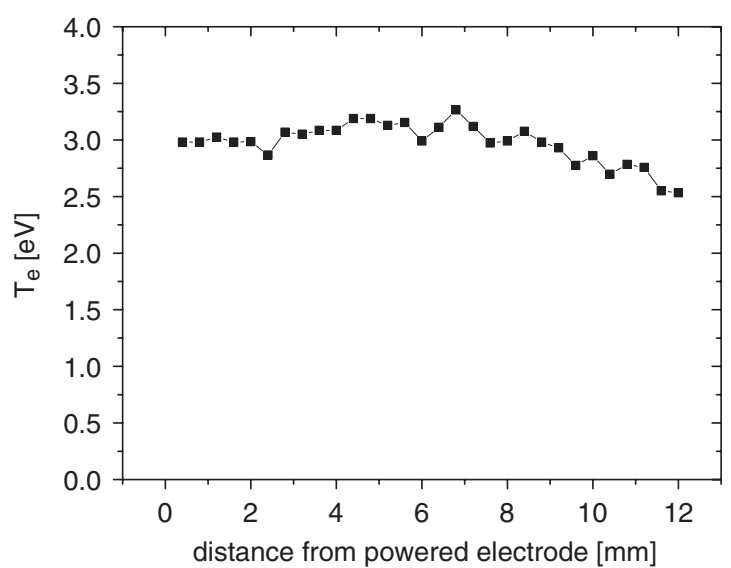

Figure 15. Space resolved electron temperature during the first half of one low frequency RF cycle.

Second, principally electron beams are also generated by the expanding high frequency sheath at phases of expanded low frequency sheath. However, this generation is less effective, since the high frequency sheath oscillates in a region of high ion density. Therefore, at a given voltage the amplitude of the high frequency sheath oscillation is low at high ion densities compared with regions of low ion densities (collapsed low frequency sheath). Consequently, electrons gain less energy at the grounded electrode sheath during the high frequency sheath expansion phase and during the first half of one $2 \mathrm{MHz}$ RF cycle. Therefore, these beams lead to much less excitation. Nevertheless, they lead to an increased electron density at one electrode, if the low frequency sheath is fully expanded. As the energy of these beam electrons is much lower, they do not propagate through the entire discharge and do not contribute to the electron density at the opposite electrode, where the highly energetic beam originates from. Third, at the phases of maximum low frequency sheath expansion secondary electrons are expected to play an important role, since the voltage is mainly determined by the low frequency. These high energetic secondary electrons lead to an increased electron density close to the respective electrode, where and when the low frequency sheath is fully expanded.

According to Brinkmann [27] the sheath edge is defined as spatial position $s$, where the following condition is fulfilled:

$$
\int_{0}^{s} n_{\mathrm{e}}(x) \mathrm{d} x=\int_{s}^{\infty}\left(n_{i}(x)-n_{\mathrm{e}}(x)\right) \mathrm{d} x,
$$

where $x=0$ is the position of the electrode. Here the sheath width is roughly estimated from a spatio-temporal plot of the excitation (figure 4) to be the distance between electrode and the spatial position where the excitation starts to increase $(0.1 \mathrm{~cm})$. As the sheath width is very small, temporal modulations of the position of the sheath edge cannot be estimated from this plot. These modulations are assumed to follow the modulation of the applied RF voltage qualitatively.

Figures 15 and 16 show the space resolved electron temperature during the first and second half of one low frequency RF cycle under the assumption of a constant temperature during each half of one cycle at each individual position. The plots justify this assumption, as the temperatures at each position do not differ substantially during the different

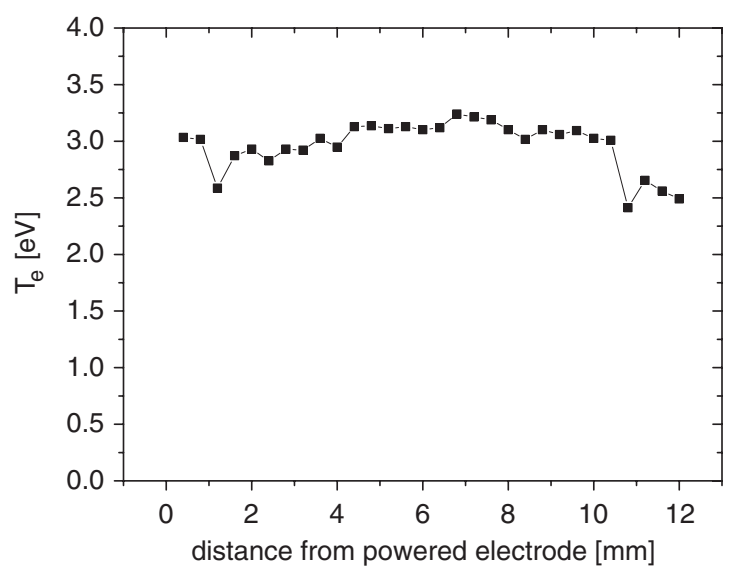

Figure 16. Space resolved electron temperature during the second half of one low frequency RF cycle.

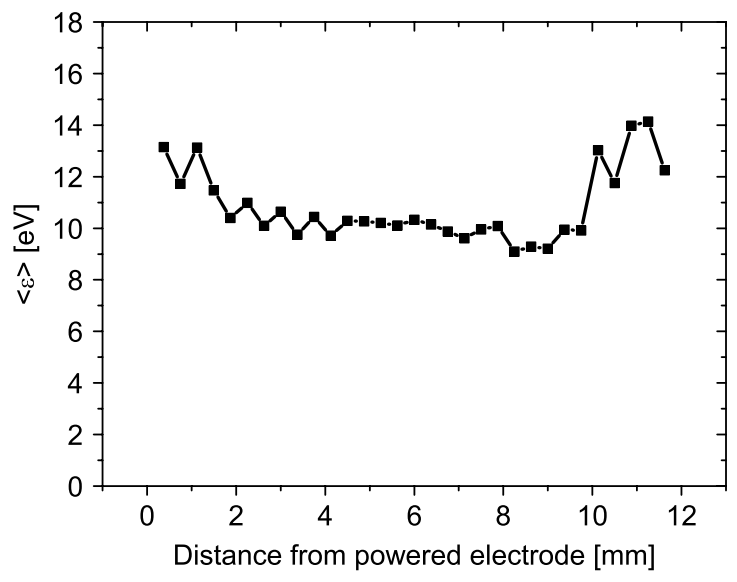

Figure 17. Space resolved electron mean energy at $86.1 \mathrm{~ns}$.

halves of one cycle. As a good approximation $T_{\mathrm{e}}$ is spatially constant. It should be noted again that $T_{\mathrm{e}}$ is not equivalent to the electron mean energy (see section 5). However, the electron mean energy $\langle\varepsilon\rangle$ at individual positions and phases can be determined from the EEDF:

$$
\langle\varepsilon\rangle=\frac{\int \varepsilon \cdot f(\varepsilon) \mathrm{d} \varepsilon}{\int f(\varepsilon) \mathrm{d} \varepsilon} .
$$

As an example the electron mean energy at $86.1 \mathrm{~ns}$ is shown spatially resolved in figure 17 . It is determined from the EEDF shown in figure 11 at a phase of maximum excitation applying equation (16). As mentioned in section 5 it is assumed, that the determined EEDF is also valid for electrons with energies below the threshold energy. However, there could be deviations, which would affect the calculated mean energy.

In contrast to $T_{\mathrm{e}}$ the electron mean energy is not spatially constant, but increases towards the electrodes, since there electrons gain energy by interacting with the sheath. In general, figures 15-17 show that the variation of the electron mean energy mainly depends on the variation of the drift velocity rather than on $T_{\mathrm{e}}$. 
Space and phase resolved plasma parameters in dual-frequency CCRF discharge

\section{Conclusions}

Important plasma parameters of an industrial dual-frequency CCRF discharge were determined with high temporal and spatial resolution. Profiles of electron temperature, drift velocity, qualitative electron density and EEDF were calculated out of the measured emission. In the corresponding algorithm a time dependent model based on rate equations is applied in order to determine the excitation functions of various excited rare gas states. Under the assumption of an adequate shape of the distribution function, that depends on the discharge geometry, the EEDF and these plasma parameters were extracted from the measured emission.

The knowledge of these plasma parameters allows a detailed analysis and understanding of electron dynamics in dual-frequency discharges. Due to the high temporal and spatial resolution of these measurements even localized phenomena within the high frequency RF cycle are observable.

Some important characteristics of electron dynamics in dual-frequency discharges could be discovered: the excitation of high energy states is strongly modulated with space and time. In particular the temporal variation is complex and depends on both frequencies. This strong coupling of both frequencies affects the excitation dynamics within the low- as well as the high frequency cycle. Therefore, the separate control of ion energy and electron density should be expected to be limited. Electron confinement by the low frequency voltage, that shields the electrodes during most of the low frequency RF cycle, might play an important role. Plasma sustainment is dominated by energetic electrons produced through the dynamics of the plasma boundary sheath. The EEDF is not purely Maxwellian at all phases, but its high energy tail is strongly modulated during most of the RF cycle. The EEDF is not shifted at phases, where the sheath edge is approximately at rest and strongly shifted to higher energies, when the sheath expands.

For industrial applications an important result is the strong coupling between both frequencies, that is most evident in the spatio-temporal excitation plots. Excitation, ionization and consequently also electron density is not determined only by the high frequency component. There is also a substantial modulation of the excitation with twice the low frequency. Furthermore, the EEDF is determined at high spatial and temporal resolution. The knowledge of the EEDF is essential for industrial processes, since it governs dissociation and ionization.

\section{Acknowledgments}

This research was supported by Lam Research Inc., the EU (FP5), the DFG (SFB 591 and GRK 1051) and the Studienstiftung des dt. Volkes. The authors would like to thank Ronan Faulkner and Paul Swift for their help with the experimental setup.

\section{Appendix}

In principle the mathematical problem solved in section 5 using the algorithm described in the paper could also be solved directly. From a purely mathematical point of view a system of three equations and three variables needs to be solved. However, this system consists of implicit functions and is, therefore, difficult to solve explicitly. If a solution is found, it will not necessarily be physically reasonable. The reason for this is the fact that the excitation is already the result of a fitting routine described in section 4 and, therefore, only an approximation.

Again from a purely mathematical point of view a function could be derived, that yields one plasma parameter in dependence of another, for example $V_{\mathrm{d}}=V_{\mathrm{d}}\left(T_{\mathrm{e}}\right)$. However, such a function does not have to be surjective. This means that not every combination of values for the excitation of the three energy levels can be reproduced. An extreme example in case of three emission lines would be the following: if lines 1 and 2 for some reason have a measured intensity of $0 \%$ at a given position and phase and line 3 an intensity of $100 \%$, there will not be a combination of $V_{\mathrm{d}}, T_{\mathrm{e}}$ and $n_{\mathrm{e}}$, that reproduces these values.

Here the problem was simplified by reducing the number of free parameters from 3 to 2 . As described before the electron temperature is determined separately at specific phases of minimum excitation, when the electron drift velocity is negligible, and assumed to be constant for one half of the low frequency RF cycle. When the electron temperature is known, the system of equations still consists of three equations (3 lines), but only two variables. Such a system of equations is overdetermined and does not have a unique solution. Therefore, the above fitting algorithm is applied minimizing the $\chi^{2}$.

\section{References}

[1] Boyle P C, Ellingboe A R and Turner M M 2004 Plasma Sources Sci. Technol. 13 493-503

[2] Boyle P C, Ellingboe A R and Turner M M 2004 J. Phys. D: Appl. Phys. 37697

[3] Kitajima T, Takeo Y, Petrovic Z L and Makabe T 2000 Appl. Phys. Lett. 77489

[4] Denda T, Miyoshi Y, Komukai Y, Goto T, Petrovic Z L and Makabe T 2004 J. Appl. Phys. 95870

[5] Kawamura E, Lieberman M A and Lichtenberg A J 2006 Phys. Plasmas 13053506

[6] Turner M M and Chabert P 2006 Phys. Rev. Lett. 96205001

[7] Gans T, Schulze J, O’Connell D, Czarnetzki U, Faulkner R, Ellingboe A R and Turner M M 2006 Appl. Phys. Lett. 89261502

[8] Karkari S K, Gaman C and Ellingboe A R 2006 Proc. 18th ESCAMPIG (Lecce)

[9] Tochikubo F, Suzuki A, Kakuta S, Terazono Y and Makabe T 1990 J. Appl. Phys. 685532

[10] Tadokoro M, Hirata H, Nakano N, Petrovic Z and Makabe T 1998 Phys. Rev. E 571

[11] Mahony C M O and Graham W G 1999 IEEE Trans. Plasma Sci. 2772

[12] Gans T, Schulz-von der Gathen V and Döbele H F 2004 Europhys. Lett. 66 232-8

[13] Gans T, Lin C C, Schulz-von der Gathen V and Döbele H F 2003 Phys. Rev. A 67012707

[14] Ellingboe A R, Boswell R W, Booth J P and Sadeghi N 1995 Phys. Plasmas 21807

[15] Gans T, Schulz-von der Gathen V and Döbele H F 2003 Proc. Frontiers in Low Temperature Plasma Physics (Bari)

[16] Niemi K, Schulz-von der Gathen V and Döbele H F 2001 J. Phys. D: Appl. Phys. 342330

[17] Francis A, Czarnetzki U, Döbele H F and Sadeghi N 1997 Appl. Phys. Lett. 713796

[18] Francis A, Czarnetzki U, Döbele H F and Sadeghi N 1998 Eur. Phys. J. 4239 
[19] NIST atomic spectra database http://physlab.nist.gov/cgi-bin/ AtData/main_asd

[20] Chilton J E, Stewart M D and Lin C C 2000 Phys. Rev. A 6152708

[21] Chilton J E, Boffard J B, Schappe R S and Lin C C 1998 Phys. Rev. A 57267

[22] Chilton J E, Stewart M D and Lin C C 2000 Phys. Rev. A 6232714
[23] Sadeghi N, Setzer D W, Francis A, Czarnetzki U and Döbele H F 2001 J. Chem. Phys. 1153144

[24] Vender D and Boswell R W 1990 IEEE Trans. Plasma Sci. 18725

[25] Wood B P 1991 PhD Thesis University of California, Berkeley

[26] O'Connell D, Gans T, Vender D, Czarnetzki U and Boswell R 2007 Phys. Plasmas 14034505

[27] Brinkmann R P 2007 J. Appl. Phys. at press 